\title{
В.Н. Трактинская
}

Днепропетровский начиональный университет

\section{ХАРАКТЕРИЗАЩИЯ ЭЛЕМЕНТА НАИЛУЧШЕГО ИНТЕГРАЛЬНОГО ПРИБЛИЖЕНИЯ ФУНКЦИЙ МНОГИХ ПЕРЕМЕННЫХ}

Отримано критерій елемента найкращого набликення для функцій багатьох змінnux у просторі $L_{p_{1}, \ldots} p_{n}$.

Пусть $\mathrm{L}_{\mathrm{p}_{1}, \ldots \mathrm{p}_{\mathrm{n}}}=\mathrm{L}_{\overline{\mathrm{p}}}\left(1 \leq \mathrm{p}_{\mathrm{i}} \leq \infty, \mathrm{i}=\overline{1, \mathrm{n}}\right)-$ пространства вещественнозначных суммируемых на $\mathrm{K}=\left[\mathrm{a}_{1}, \mathrm{~b}_{1}\right] \times \ldots \times\left[\mathrm{a}_{\mathrm{n}}, \mathrm{b}_{\mathrm{n}}\right]$ функций $\mathrm{n}$ переменных $f\left(x_{1}, x_{2}, \ldots x_{n}\right)=f(x)$ с конечной нормой

$$
\|f\|_{\bar{p}}=\|f\|_{p_{1}, \ldots p_{n}}=\left[\int_{a_{n}}^{b_{n}} \ldots\left[\int_{a_{2}}^{b_{2}}\left[\int_{a_{1}}^{b_{1}} f(x) \mid d x_{1}\right]^{\frac{p_{2}}{p_{1}}} d x_{2}\right]^{\frac{p_{3}}{p_{2}}} \ldots d x_{n}\right]^{\frac{1}{p_{n}}} .
$$

Положим

$$
|f|_{p_{k}, \ldots p_{i}}=\left[\int_{a_{i}}^{b_{i}}\left[\int_{a_{k+1}}^{b_{k+1}}\left[\int_{a_{k}}^{b_{k}}|f(x)|^{p_{k}} d x_{k}\right]^{\frac{p_{k+1}}{p_{k}}} d x_{2}\right]^{\frac{p_{k+2}}{p_{k+1}}} \ldots d x_{i}\right]^{\frac{1}{p_{i}}},
$$

где $1 \leq \mathrm{k}<\mathrm{n}, 1<\mathrm{i} \leq \mathrm{n}$.

Введем также в рассмотрение классы $L_{q_{1}, \ldots q_{n}}$ (где хотя бы одно $q_{i}=\infty$ ) функций $\mathrm{f}$, нормы которых определяются по формулам:

$$
\begin{aligned}
& \|f\|_{p_{1}, \ldots p_{n-1}, \infty}=\underset{x_{n} \in\left[a_{n}, b_{n}\right]}{\operatorname{esssup}}|f|_{p_{1}, \ldots p_{n-1}}, \\
& \|f\|_{p_{1}, \ldots, p_{i-1}, \infty, p_{i+1}, \ldots p_{n}}=\left[\int_{a_{n}}^{b_{n}}\left[\int_{a_{i+1}}^{b_{i+1}}\left({\left.\left.\operatorname{ess~sup~}|f|_{p_{i+1}, \ldots p_{n}}\right)^{p_{i+1}} d a_{i}, b_{i}\right]}_{p_{i+1}}\right]^{\frac{p_{i+2}}{p_{i+1}}} \ldots d x_{n}\right]^{\frac{1}{p_{n}}},\right.
\end{aligned}
$$

где $1 \leq \mathrm{i}<\mathrm{n}$, и конечны.

Г.С. Смирновым [1] был установлен критерий элемента наилучшего приближения в пространствах $L_{p, q}(K)$, где $K=[a, b] \times[c, d]$. Цель настоящей рабо- 
ты - распространение результата Г.С. Смирнова на случай приближения функций $\mathrm{n}(\mathrm{n} \geq 2)$ переменных.

Заметим, что для любых пар функций $f \in L_{\bar{p}}$ и $q \in L_{\bar{q}}$ $\left(\frac{1}{p_{i}}+\frac{1}{q_{i}}=1, i=\overline{1, n}\right)$ имеет место обобщение неравенства Гельдера:

$$
\left|\int_{\mathrm{K}} \mathrm{f}(\mathrm{x}) \varphi(\mathrm{x}) \mathrm{dx}_{1} \ldots \mathrm{dx} \mathrm{x}_{\mathrm{n}}\right| \leq\|\mathrm{f}\|_{\overline{\mathrm{p}}}\|\varphi\|_{\overline{\mathrm{q}}} .
$$

Повторяя рассуждения соответствующей теоремы о виде линейного функционала, несложно получить следующее утверждение.

Теорема 1. Всякий линейный непрерывный функиионал, заданный на пространстве $\mathrm{L}_{\overline{\mathrm{p}}}$ ииеет вид $\mathrm{F}(\mathrm{f})=\int_{\mathrm{K}} \mathrm{f}(\mathrm{x}) \alpha(\mathrm{x}) \mathrm{dx}_{1} \ldots \mathrm{dx}_{\mathrm{n}}$, где $\mathrm{f}(\mathrm{x})-$ произвольная функиия из $\mathrm{L}_{\overline{\mathrm{p}}}$, a $\alpha(\mathrm{x})$ - некоторая функиия из $\mathrm{L}_{\overline{\mathrm{q}}}$, определяемая по функционалу $\mathrm{F}$, и при этом $\|\mathrm{F}\|=\|\alpha\|_{\bar{q}}$.

Пусть на $\mathrm{K}$ задана линейно независимая система функций $\varphi_{\mathrm{k}} \in \mathrm{L}_{\overline{\mathrm{p}}}$ $(\mathrm{k}=\overline{1, \mathrm{~m}})$. Обозначим $\mathrm{H}_{\mathrm{m}}=\operatorname{span}\left\{\varphi_{1}, \ldots \varphi_{\mathrm{m}}\right\}, \mathrm{E}\left(\mathrm{f}, \mathrm{H}_{\mathrm{m}}\right)_{\overline{\mathrm{p}}}=\mathrm{E}_{\mathrm{m}}(\mathrm{f})_{\overline{\mathrm{p}}} \cdot \mathrm{B}$ дальнейшем нам понадобятся следующие утверждения

Лемма. Если $\mathrm{f} \in \mathrm{L}_{\overline{\mathrm{p}}}$, то

$$
\|\mathrm{f}\|_{\overline{\mathrm{p}}}=\sup _{\mathrm{g}} \int_{\mathrm{K}} \mathrm{f}(\mathrm{x}) \mathrm{g}(\mathrm{x}) \mathrm{d} \mathrm{x}_{1} \ldots \mathrm{d} \mathrm{x}_{\mathrm{n}},
$$

где sup распространен на всевозможные функиии $\mathrm{g} \in \mathrm{L}_{\bar{q}},\|\mathrm{~g}\|_{\bar{q}} \leq 1$.

Если $\|\mathrm{f}\|_{\bar{p}}>0$, то sup в правой части (1) достигается для функции вида

$$
\mathrm{g}_{0}(\mathrm{x})=\left\{\begin{array}{l}
|\mathrm{f}|^{\mathrm{p}_{1}-1}|\mathrm{f}|_{\mathrm{p}_{1}}^{\mathrm{p}_{2}-\mathrm{p}_{1}} \ldots|\mathrm{f}|_{\mathrm{p}_{1}, \ldots, \mathrm{p}_{\mathrm{n}-1}}^{\mathrm{p}_{\mathrm{n}}-\mathrm{p}_{\mathrm{n}-1}} \|\left.\mathrm{f}\right|_{\overline{\mathrm{p}}} ^{1-\mathrm{p}_{\mathrm{n}}} \operatorname{sgn} \mathrm{f}, \quad \text { если }|\mathrm{f}|_{\mathrm{p}_{1}, \ldots, \mathrm{p}_{\mathrm{n}-1}} \neq 0, \\
0, \quad \text { если }|\mathrm{f}|_{\mathrm{p}_{1}, \ldots, \mathrm{p}_{\mathrm{n}-1}}=0 .
\end{array}\right.
$$

Функция $\mathrm{g}_{0}$ будет единственной (если хотя бы одно из $\mathrm{p}_{\mathrm{i}}=1$, в предположении, что $\mathrm{f}(\mathrm{x}) \neq 0$ почти всюду на $\mathrm{K})$.

Утверждение 1. Для любой функции $\mathrm{f} \in \mathrm{L}_{\overline{\mathrm{p}}}$

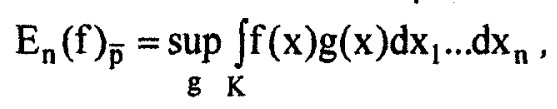

где sup распространен на функиии $\mathrm{g} \in \mathrm{L}_{\bar{q}}$, такие что $\|\mathrm{g}\|_{\bar{q}} \leq 1 \cdot u \mathrm{~g} \perp \mathrm{H}_{\mathrm{m}}$. Sup 6 (2) достигается на некоторых функииях $\varphi_{0} \in \mathrm{L}_{\bar{q}}$ с нормой $\left\|\varphi_{0}\right\|_{\bar{q}}=1$.

Утверждение 2. Полином $\mathrm{P}_{\mathrm{m}}^{*} \in \mathrm{H}_{\mathfrak{m}}$ является элементом наилучшего приближения для $\mathrm{f} \in \mathrm{L}_{\overline{\mathrm{p}}} \backslash \mathrm{H}_{\mathrm{m}}$ тогда и только тогда, когда существует функчия $\mathrm{g}_{0} \in \mathrm{L}_{\overline{\mathrm{q}}}$, удовлетворяющая условиям:

1) $\left\|g_{0}\right\|_{\bar{q}}=1$; 
2) $\|f\|_{\bar{p}}=\int_{K} f(x) g_{0}(x) d x_{1} \ldots d x_{n}$;

3) $\int_{\mathrm{K}} \mathrm{P}_{\mathrm{m}}(\mathrm{x}) \mathrm{g}_{0}(\mathrm{x}) \mathrm{dx_{1 }} \ldots \mathrm{d} \mathrm{x}_{\mathrm{n}}=0, \quad \forall \mathrm{P}_{\mathrm{m}} \in \mathrm{H}_{\mathrm{m}}$.

Теорема 2. Для того, чтобы полином $\mathrm{P}_{\mathrm{m}}^{*} \in \mathrm{H}_{\mathrm{m}}$ был элементом наилучшего приближения для функции $\mathrm{f} \notin \mathrm{H}_{\mathrm{m}}$ достаточно и (если хотя бы одно из $\mathrm{p}_{\mathrm{i}}=1$ в случае, когда $\mathrm{f}-\mathrm{P}_{\mathrm{m}}^{*} \neq 0$ почти всюоу на $\mathrm{K}$ ) необходимо выполнение соотночения $\int_{\mathrm{K}} \mathrm{P}_{\mathrm{m}}(\mathrm{x}) \mathrm{g}(\mathrm{x}) \mathrm{dx}_{1} \ldots \mathrm{dx}_{\mathrm{n}}=0, \forall \mathrm{P}_{\mathrm{m}} \in \mathrm{H}_{\mathrm{m}}$, где

$$
g(x)=\left\{\begin{array}{l}
\left|f-P_{m}^{*}\right|^{p_{1}-1}\left|f-P_{m}^{*}\right|_{p_{1}}^{p_{2}-p_{1}} \ldots\left|f-P_{m}^{*}\right|_{p_{1}, \ldots, p_{n-1}}^{p_{n}-p_{n-1}} \operatorname{sgn}\left(f-P_{m}^{*}\right), \text { если }\left|f-P_{m}^{*}\right|_{p_{1}, \ldots, p_{n-1}} \neq 0, \\
0, \text { если }\left|f-P_{m}^{*}\right|_{p_{1}, \ldots, p_{n-1}}=0 .
\end{array}\right.
$$

Доказательство. Достаточность. Очевидно, что $\|\mathrm{g}\|_{\bar{q}}=\left\|f-P_{m}^{*}\right\|_{\bar{p}}^{p_{n}-1}$. Тогда $\int_{\mathrm{K}} \mathrm{f}(\mathrm{x}) \mathrm{g}(\mathrm{x}) \mathrm{dx} \mathrm{x}_{1} \ldots \mathrm{dx_{ \textrm {n } }}=\int_{\mathrm{K}}\left(\mathrm{f}-\mathrm{P}_{\mathrm{m}}^{*}\right)(\mathrm{x}) \mathrm{g}(\mathrm{x}) \mathrm{dx} \mathrm{x}_{1} \ldots \mathrm{dx} \mathrm{x}_{\mathrm{n}}=\left\|\mathrm{f}-\mathrm{P}_{\mathrm{m}}^{*}\right\|_{\bar{p}}^{\mathrm{P}_{\mathrm{n}}}$. . С другой стороны, из утверждения 1 следует, что $\int_{\mathrm{K}} \mathrm{f}(\mathrm{x}) \mathrm{g}(\mathrm{x}) \mathrm{dx} \mathrm{x}_{1} \ldots \mathrm{dx}_{\mathrm{n}} \leq \mathrm{E}_{\mathrm{n}}(\mathrm{f})_{\overline{\mathrm{p}}} \cdot\left\|\mathrm{f}-\mathrm{P}_{\mathrm{m}}^{*}\right\|_{\overline{\mathrm{p}}}^{\mathrm{p}_{\mathrm{n}}-1}$. Тогда получаем: $\left\|f-P_{m}^{*}\right\|_{\bar{p}} \leq E_{n}(f)_{\bar{p}}$.

Необходимость. Пусть $\mathrm{P}_{\mathrm{n}}^{*}$ - полином наилучшего приближения для функции $f \in \mathrm{L}_{\overline{\mathrm{p}}}$. Тогда в силу утверждения 2 , найдется функция $\mathrm{g}_{0} \in \mathrm{L}_{\overline{\mathbf{q}}}$, удовлетворяющая условиям (3). В силу леммы условие (2) будет выполняться для функции $\mathrm{g}_{0}(\mathrm{x})$, равной

$$
\left|f-P_{m}^{*}\right|^{p_{1}-1}\left|f-P_{m}^{*}\right|_{p_{1}}^{p_{2}-p_{1}} \ldots\left|f-P_{m}^{*}\right|_{p_{1}, \ldots, p_{n-1}}^{p_{n}-p_{n-1}} \| f-\left.P_{m}^{*}\right|_{\bar{p}} ^{1-p_{n}} \operatorname{sgn}\left(f-P_{m}^{*}\right),
$$

если $\left|\mathrm{f}-\mathrm{P}_{\mathrm{m}}^{*}\right|_{\mathrm{p}_{1}, \ldots, \mathrm{p}_{\mathrm{n}-1}} \neq 0$, и равной 0 , если $\left|\mathrm{f}-\mathrm{P}_{\mathrm{m}}^{*}\right|_{\mathrm{p}_{1}, \ldots, \mathrm{p}_{\mathrm{n}-1}}=0$, причем функция $\mathrm{g}_{0}$ будет единственной. Но тогда из условия 3 утверждения 2 получаем: $\int_{\mathrm{K}} \mathrm{P}_{\mathrm{m}}(\mathrm{x}) \mathrm{g}(\mathrm{x}) \mathrm{dx} \mathrm{x}_{1} \ldots \mathrm{dx} \mathrm{x}_{\mathrm{n}}=0, \forall \mathrm{P}_{\mathrm{m}} \in \mathrm{H}_{\mathrm{m}}$.

Теорема 2 полностью доказана.

1. Смирнов Г. С. Общий вид линейного функционала и критерий полинома наилучшего приближения в пространствах со смешанной интегральной метрикой// Укр. мат. журн. 1973. - Т.25, № 1. С. $134-138$.

Надійила до редколегї 25.03.07 